\title{
Measurement problem of structural-parametric identification on supernovae type SN Ia for cosmological distances scale of red shift based
}

\section{Levin S.F.}

Moscow Institute for expertise and tests, Department of metrology and metrological support, Moscow, Russia; E-mail: Levin<AntoninaEL@rostest.ru>;

By data, on which basis within the limits of model Friedman-Robertson-Walker the conclusion has been drawn on the beginning of «accelerated expansion of Universe» about 6 billion years ago, the problem of calibration for interpolation models of distances scale with form parameter is solved. According to results of identification at them Doppler interpretations in «Universe expansion» about 2.64-3.18 billion years ago there was pause. The pause was replaced by accelerated expansion which by modern epoch has degenerated in Hubble's stream. On similar data of Sternberg Institute the interpolation model gives practically linear dependence of red shift on photometric distance.

Keywords: cosmological distances scale, interpolation model with form parameter, structurally-parametrical identification, «magnitude standard» of supernovae type SN Ia.

DOI: $10.18698 / 2309-7604-2015-1-299-310$

\section{Introduction}

The major metrological designs in astronomy and cosmology are scales of distances $D$. All of them are based on method of indirect measurement [1]. The equation of method of indirect measurement of distance or the equation of distances scale can be based on known geometrical parities, physical laws or on likelihood models of stochastic dependences between distance to observable object and the measured physical sizes connected with it.

Presence in radiation spectra of the majority of extragalactic objects of red shift $z$ has generated on this basis a number of cosmological distances scales. Red shift is accessible to measurement at identification issue or absorptive lines as part of spectrum by comparison with set of spectral lines in terrestrial conditions.

The physical nature of red shift is connected with gravitational shift $z_{\mathrm{g}}$ at the expense of difference of gravitational potentials in radiation and reception points and Doppler shift $z_{\mathrm{v}}$ at the expense of movement of source of radiation concerning point of reception [2].

Besides, by analogy to «dark matter» and «dark energy» it is possible to assume existence of one more mechanism of red shift - «dark attenuation». From this point of view to cosmological red shift $z_{\kappa}$ we will carry its component not identified on a source.

Distances scales $D_{Z}(z)$ on the basis of red shift are the kind equations 


$$
\begin{aligned}
& D_{Z}(z)=\left(c / H_{0}\right) \cdot z \\
& D_{Z}(z)=\left(c / H_{0}\right) \cdot\left[(1+z)^{2}-1\right] /\left[(1+z)^{2}+1\right], \\
& D_{Z}(z)=\left(R_{0} / q_{0}^{2}\right) \cdot\left[q_{0} z+\left(q_{0}-1\right) \cdot\left(\sqrt{2 q_{0} z+1}-1\right)\right] \\
& D_{Z}(z)=(2 K)^{-1} \cdot\left[\sqrt{H_{0}^{2}+4 K \cdot c \cdot z}-H_{0}\right] \text {, } \\
& D_{Z}(z)=\left(c / H_{0}\right) \cdot[z /(1+z)] \\
& D_{z}(z)=\left(R_{0} / k\right) \cdot\left[(1+z)^{k}-1\right] \text { or }\left(D_{z} / R_{0}\right)^{k}+k \cdot\left(D_{z} / R_{0}\right) \cdot z^{k}=z^{k} \\
& D_{Z}=\frac{c \cdot(1+z)}{H_{0} \cdot\left|\Omega_{k}\right|^{1 / 2}} \cdot \operatorname{Six}\left\{\left|\Omega_{k}\right|^{1 / 2} \int_{0}^{Z}\left[(1+Z)^{2}\left(1+\Omega_{M} \cdot Z\right)-Z(2+Z) \cdot \Omega_{\Lambda}\right]^{-1 / 2} d z\right\} \\
& \operatorname{Six}\{\cdot\}=\left\{\begin{array}{l}
\operatorname{sh}\{\cdot\}, \Omega_{k} \geq 0 \\
\sin \{\cdot\}, \Omega_{k} \leq 0
\end{array}\right.
\end{aligned}
$$

where $c$ - fundamental constant of light velocity, $H_{0}$ - Hubble's constant, $R_{0}=c / H_{0}-$ Hubble's radius, $q_{0}$ - delay/acceleration parameter, $K$ - Hoyle's parameter, $k$ - form parameter, $\Omega_{M}$ - density of weights, $\Omega_{\Lambda}$ - density of «dark energy», $\Omega_{k}=1-\Omega_{M}-\Omega_{\Lambda}$.

Hubble's scale (1) [3], it Doppler variant (2) and scale on the basis of kinematical model (5) [4] actually contain one parameter $R_{0}=c / H_{0}$, and accuracy of these scales is defined by accuracy of estimation of Hubble's constant. Mattig's scale (3) [5], Hoyle's scale (4) [6] and interpolation scales (6) [4] have on one additional parameter, and scale in model FriedmanRobertson-Walker (7) [7] - two additional parameters. Also there is natural question, whether gives this circumstance and additional possibilities on increase in accuracy of these scales at the expense of calibration on these parameters? 


\section{Problems of calibration of scales cosmological distances on red shift}

Structurally-parametrical identification of mathematical models of measurements objects within the limits of method of collateral measurements [1] is spent by criterion of minimum accepted functional inadequacy errors, for example, the average module of casual component. Before introduction [1] problem here was that an inadequacy error named an approximation error of model for measurements data.

This mess at number increase $n$ model parameters conducted to reduction of an error of approximation, and at equality of number of parameters of model to volume of sample of the given measurements the approximation error appeared equal to zero. But at increase in sample at unit without recalculation of parameters at the expense of inadequacy of model this readout, as a rule, turned to «allocated result» or «rough error».

The minimum of inadequacy error of model of physical object is reached at equality of structural and parametrical components, and its position on an axis of structure codes of models depends on dimensional component [1]. To more difficult models there correspond more exact measurements. This minimum corresponds to terminological phrase - «in the ideal image in the qualitative and quantitative relation».

Calibration of scale (7) on reference points of photometric distances scale $D_{L}$ with standard $M_{\text {st SN Ia }}=-19,37^{m}$ absolute magnitude of supernovae type SN Ia has allowed to receive fundamental result to Big Bang theory [8, 9]: «Universe expansion occurs to acceleration».

Actually it means recognition of method for definition cosmological distances under standard $M_{\text {st SN Ia }}$ as the fundamental. To this circumstance it is necessary to add necessarily the result established during experiment WMAP [10]: global geometry of astronomical Universe practically «flat» (Euclidian) with parameter of spatial curvature $\Omega_{k}=-0,0027^{+0,0039} /{ }_{-0,0038}$.

Standard $M_{\text {st SN Ia }}$ is based that in double system the white dwarf, reaching on weight of Chandrasekhar limit [11] for the account accretion substances of the companion, becomes supernovae $(\mathrm{SN})$ from almost constant luminosity in maximum in absolute magnitude. Distance modules of reference points for this scale $\mu_{0}=m^{\text {peak }}-M_{\mathrm{stSN} \mathrm{Ia}}=5 \cdot \lg D_{L}+25$ establish method of collateral measurements under Hubble's diagram, under forms or templates of curves of luminosity introduction of amendments for current observable magnitude for luminosity maximum $m^{\text {peak }}$.

For «flat» cosmology the best approximation by the form of curve luminosity method has given $\left(\Omega_{M}=0,24 ; \Omega_{\Lambda}=0,76\right)$, template method $-\left(\Omega_{M}=0,20 ; \Omega_{\Lambda}=0,80\right)$ [8] and method of the Hubble's diagram $-\left(\Omega_{M}=0,28 ; \Omega_{\Lambda}=0,72\right)$ [9], that corresponds $\Omega_{k}=0$.

Calibration of such scales of distances has number of the problem moments. 
First, shift in spectra can contain little making and depending on their parity can be red or violet: $z=\left(1+z_{g}\right)\left(1+z_{\mathrm{v}}\right)\left(1+z_{\mathrm{K}}\right)-1$. For gravitational component $z_{g}=\sqrt{\left(1-2 \varphi_{0} / c^{2}\right) /\left(1-2 \varphi_{e} / c^{2}\right)}-1$, where $\varphi_{e}$ and $\varphi_{0}$ - gravitational potentials in points accordingly radiations and measurements, usually neglect, as gravitational red shift of white dwarfs makes $z_{g} \leq 10^{-3}$. And though by the flash moment gravitational red shift of the white dwarf increases more than 10 times, it masks Doppler violet shift at the expense of extending at explosion towards the observer of cover of the white dwarf with speed of an order $3 \cdot 10^{4} \mathrm{~km} \cdot \mathrm{s}^{-1}$. Doppler component of red shift $z_{\mathrm{v}}=\left(1-\mathrm{V}_{r} / c\right) / \sqrt{1-\left(\mathrm{V}_{r}^{2}+\mathrm{V}_{t g}^{2}\right) / c^{2}}-1$, where $\mathrm{V}_{r}$ and $\mathrm{V}_{t g}$ - radial and tangential component of movement speed of object concerning the observer. But in cosmological models «Universe expansions» divide it on peculiar and cosmological components. The first of them at big red shift neglect, and last believe Doppler and connect with «dark energy».

Alternative to «dark» factors is «dark attenuation».

As result red shift into components do not divide, and for SN accept red shift of host galaxies, as took place in $[8,9]$.

Secondly, calibration for distances scale assumes presence of quantity of reference points which characteristics are known with accuracy, obviously not below demanded accuracy of calibration. Such characteristic is mathematical model of dependence on observable sizes of estimations for red shift not so much actually SN, how many red shift of their host galaxies:

$$
Z \Leftrightarrow D_{L}=10^{-5+0,2\left(m^{\text {peak }}-M_{\text {stSNla }}\right)}=10^{-5+0,2 \mu_{0}},
$$

where standard deviations of estimations for red shift make $\sigma_{z} \sim 10^{-3}[9]$.

In [8] set of reference points sample from $27 \mathrm{SN}$ forms at $z<0,13$ and $10 \mathrm{SN}$ at $0,30 \leq z \leq$ 0,97 , and in [9] - sample from $42 \mathrm{SN}$ with red shift $0,354 \leq z \leq 0,828$. The standard of absolute luminosity $M_{\text {st SN Ia }}=-19,37^{m}$ is accepted on SN $1992 \mathrm{bs}\left(z=0,063 ; m_{B}^{\text {peak }}=18,24 ; \mu_{0}=37,6^{m}\right)$ and SN 1997ap $\left(z=0,830 ; m_{B}^{\text {peak }}=24,30 ; \mu_{0}=43,67^{m}\right)$ in filter $B$. For others $\mathrm{SN}$ in [8] deviations from standard $M_{\text {st SN Ia }}=-19,37^{m}$ are limited by an interval $\left[-0,52^{m} ;+0,40^{m}\right]$, and in [9] residual deviations of effective observable star sizes on Hubble's diagram are limited by interval $\left[-0,7^{m}\right.$; $+1,3^{m}$ ]. However, in [9] SN 1992bs in sample has not entered; it is mentioned in review [12].

In [9] transition from delay to «accelerated expansion of Universe» it is dated by flash SN $1997 \mathrm{G}\left(m_{B}^{\text {peak }}=24,49^{m} ; z=0,763\right) \sim 6 \cdot 10^{9}$ years back. 
To this moment in «flat» cosmology corresponds $D_{L} \approx 1842 \mathrm{Mpc}$, but thus $M_{\mathrm{st}} \mathrm{SN}$ Ia $=-$ $16,84^{m}$.

The deviation from the standard of absolute luminosity $2,53^{m}$ leaves far beyond the specified deviations. However, dating in [9] is connected with scale of photometric distances of type «Hubble-constant-free», in detail enough quantitatively not described.

However among so-called «unexpected coincidence» [13] maximum of acceleration equivalent on Doppler effect «Universe expansions» on scale (5) $\mathrm{w}_{\text {макс }}=9,59 \cdot 10^{-10} \mathrm{~m} \cdot \mathrm{s}^{-2}$ are necessary on $z=0,732$ and $D_{L} \approx 1667,2 \mathrm{Mpc}=5,4$ billion light years, and to scale zero-point strictly there corresponds acceleration $\mathrm{w}(0)=c \cdot H_{0}=7,21 \cdot 10^{-10} \mathrm{~m} \cdot \mathrm{s}^{-2}$ [11]. This estimation coincides with an abnormal component of acceleration Pioneer-10 on $23^{\text {rd }}$ year of flight. However problem of other scales that at similar Doppler interpretations for them $\mathrm{w}(0)=0$ and, the most important thing, «anomaly of Pioneers» corresponds not red, but to violet shift.

Thirdly, the decision of considered problem of calibration by method of collateral measurements demands the account of inadequacy errors of interpreting model [1] in common with statistical variability of measurements data, i.e. not only kind of probabilities distribution, and compositions of distributions within the limits of this model. The best fitting for «flat» cosmology is reached at $\Omega_{M}=0,24$ and $\Omega_{\Lambda}=0,76$ in [8] and at $\Omega_{M}=0,28$ and $\Omega_{\Lambda}=0,72$ in [9].

In these cases $\Omega_{M}+\Omega_{\Lambda}=1$ or $\Omega_{k}=0$, also becomes record of the formula appreciable carelessness (7) in [8] at $\Omega_{k}=0$. In [8] for peculiar making beam speed at dispersion of estimations in limits $100 \mathrm{~km} \cdot \mathrm{s}^{-1} \leq \sigma_{v} \leq 400 \mathrm{~km} \cdot \mathrm{s}^{-1}$ standard uncertainty or mean square deviation $\sigma_{v} \sim 200 \mathrm{~km} \cdot \mathrm{s}^{-}$ , and in [9] $-\sigma_{v} \sim 300 \mathrm{~km} \cdot \mathrm{s}^{-1}$ or $\sim 10^{-3}$ in units $z$ has been accepted. At the same time received in $[8,9]$ results were limited to consideration of this problem moment of calibration only concerning so-called «normal law» or Gauss distribution. Check according to [15] on number of distributions, including truncated, has shown, that is essential probability maximum of the consent to approximation errors of model in [8] truncated Laplace distribution is. In border of the maximum likelihood method in [8] used an estimation of dispersion parameter for this distribution is not mean square deviation, and the average absolute deviation [16].

Fourthly, thanks to that for Hoyle's scale $K \approx c \cdot\left(H_{0} / c\right)^{2}$, in [4] has been shown that for the description of red shift can be involved as physical, so and interpolation models. 


\section{Calibration for interpolation cosmological distances scales}

Let's consider measuring problem of calibration for cosmological distances scale (6) according to [9] about red displacement $z$ and effective star sizes 42 supernovae SN Ia on maximum of shine $m_{B}^{\text {eff }}$ (Table 3 ) for model of red shift [4]

$$
z_{k}=\left(D_{L} / R_{0}\right)\left(1-k D_{L} / R_{0}\right)^{-1 / k}
$$

For comparison data [17] (Table 1) in which beam speed is defined as $\mathrm{V}_{r}=c \cdot z$, and photographic magnitude $m$ are used specified with color index.

Table 1. Reference points for interpolation scales at $H_{0}=74,2 \mathrm{~km} \cdot \mathrm{c}^{-1} \cdot \mathrm{Mpc}^{-1}$ and $M_{\mathrm{st}} \mathrm{SN}$ Ia $=-$ $16,84^{m}$

\begin{tabular}{|c|c|c|c|c|c|c|c|c|c|c|c|c|c|c|}
\hline \multirow{2}{*}{ SN } & \multicolumn{2}{|c|}{ Data [9] } & \multicolumn{2}{|c|}{ Data [17] } & \multirow{2}{*}{ SN } & \multicolumn{2}{|c|}{ Data [9] } & \multicolumn{2}{|c|}{ Data [17] } & \multirow{2}{*}{ SN } & \multicolumn{2}{|c|}{ Data 9] } & \multicolumn{2}{|c|}{ Data [17] } \\
\hline & $m_{B}^{\mathrm{eff}}$ & $z n$ & $m$ & & & $m_{B}^{\mathrm{eff}}$ & $z n$ & $z n$ & & & $m_{B}^{\mathrm{eff}}$ & $z n$ & $z n$ & $\mathrm{~V}_{r}, \mathrm{~km} \cdot \mathrm{c}^{-}$ \\
\hline $1992 \mathrm{bi}$ & 23,11 & 0,458 & 22 & 05 & $\mathrm{Z}$ & 22,51 & 0 & R 24 & & $\bar{K}$ & 24,42 & 0,592 &, 6 & 6878 \\
\hline $1994 \mathrm{~F}$ & 2,38 & 0,354 & $>\mathrm{R} 22$ & 26 & $5 \mathrm{ba}$ & 22,65 & 0,388 & 2 & 19 & $97 \mathrm{~L}$ & 23,51 & 50 & 1 & Th \\
\hline 1994G & 22,13 & 0,425 & $>\mathrm{I} 21,8$ & 1411 & $1780 \mathrm{~s}$ & 23,27 & 0,570 & $>\mathrm{R} 22,7$ & 70882 & $1997 \mathrm{~N}$ & 20,43 & 0,180 & $\|<1,2$ & 3963 \\
\hline $994 \mathrm{H}$ & 21,72 & 0,374 & R 21,9 & 212 & $1996 \mathrm{cg}$ & 23,10 & 0,490 & $>$ R 22,5 & & 19970 & 23,52 & 0,374 & 23,7 & 110923 \\
\hline$\overline{94}$ & ,55 & 20 & 6 & 125913 & 1996ci & 33 & 5 & 3 & 148397 & $\overline{\mathbf{P}}$ & 3 , & 2 & 2 & 1902 \\
\hline $94 \mathrm{am}$ & 22,26 & 0,372 & 21,7 & 523 & 1996ck & 23,57 & 0,656 & $>$ R 23 & 64 & 199 & 22,57 & 0,430 & $>22,5$ & 131909 \\
\hline 1994an & 22,58 & 0,378 & $>22,3$ & 322 & $1996 \mathrm{cl}$ & 24,65 & 0,828 & $>\mathrm{I} 23,5$ & 248228 & $\mid$\begin{tabular}{|l|}
$1997 R$ \\
\end{tabular} & 23,83 & 0,657 & $>24,4$ & 194865 \\
\hline 1995aq & 23,17 & 0,453 & $>$ R 22,4 & 135805 & $1996 \mathrm{~cm}$ & 23,17 & 0,450 & $>\mathrm{R} 22,7$ & 4907 & $997 \mathrm{~S}$ & 23,69 & 0,612 & $>23,6$ & 2873 \\
\hline 1995ar & 23,33 & 465 & $>$ R 23,1 & 1 & n & 23 & 0 & -P? & 1 & $\mathrm{c}$ & 2 & 0,320 & 72 & 05034 \\
\hline 1995as & 23,71 & 0,498 & $>$ R 23,3 & 149296 & 1997F & 23,46 & 0,580 & $>23,9$ & 880 & \begin{tabular}{|l|l|} 
1997af \\
\end{tabular} & 23,48 & 0,579 & $>22,3$ & 173580 \\
\hline 1995at & 23,27 & 0,655 & $>\mathrm{R} 22,7$ & 19 & G & 24,47 & 0,763 & 23,7 & $H 1$ & ai & 22,83 & 0,450 & 22,3 & 13 \\
\hline 1995aw & 22,36 & 0,400 & $>\mathrm{R} 22,5$ & 119917 & $1997 \mathrm{H}$ & 23,15 & 0,526 & $>22,8$ & 8890 & 1997aj & 23,09 & 0,581 & $>23,8$ & 174179 \\
\hline 1995ax & 23,19 & 0,615 & $>$ R 22,6 & 184372 & 1997I & 20,17 & 0,172 & $\mid>20,9$ & 53963 & 1997am & 22,57 & 0,416 & $>22,9$ & 124714 \\
\hline 1995ay & 22,96 & 0,480 & $>$ R 22,7 & 143900 & 1997J & 23,80 & 0,619 & $>23,4$ & 185571 & 1997ap & 24,32 & 0,830 & $>24,2$ & 248828 \\
\hline
\end{tabular}

Model (8) has been checked up on parameterization correctness by substitution of distances module and decision of equations system concerning of form parameter (Table 2)

$$
Z_{\text {к }}=\left(R_{0}^{-1} 10^{-5+0,2\left(m_{B, n}^{\text {eff }}-M_{\text {stSNla }}\right)}\right)\left(1-k_{n} R_{0}^{-1} 10^{-5+0,2\left(m_{B, n}^{\text {eff }}-M_{s t S N l a}\right)}\right)^{-1 / k_{n}}, \quad n=\overline{1,42} .
$$


Table 2. Form parameter of cosmological distances scale

\begin{tabular}{|c|c|c|c|c|c|c|c|c|c|}
\hline \multirow{2}{*}{ SN } & \multicolumn{2}{|c|}{ Data [9] } & \multicolumn{2}{|c|}{ Data [17] } & \multirow{2}{*}{ SN } & \multicolumn{2}{|c|}{ Data [9] } & \multicolumn{2}{|c|}{ Data [17] } \\
\hline & $D_{L}, \mathrm{Mpc}$ & $k_{n}$ & $D_{L}, \mathrm{I}$ & $k_{n}$ & & $D_{L}, \mathrm{\Lambda}$ & $k_{n}$ & $D_{L}$, & $k_{n}$ \\
\hline 1992bi & \begin{tabular}{|l|}
977,237 \\
\end{tabular} & 3,75948 & 586,138 & 6,89063 & $1996 \mathrm{~cm}$ & 1004,616 & 3,52480 & 809,096 & 4,89906 \\
\hline 1994F & \begin{tabular}{|l|}
698,232 \\
\end{tabular} & 5,68859 & \begin{tabular}{|c|}
586,138 \\
\end{tabular} & 6,87822 & $1996 \mathrm{cn}$ & 86,279 & 3,54651 & \begin{tabular}{|l|}
772,681 \\
\end{tabular} & 5,14829 \\
\hline 1994G & 2,300 & 6,48357 & 64 & 55705 & $1997 \mathrm{~F}$ & 48,154 & 3,14605 & 48 & 1,61280 \\
\hline $1994 \mathrm{H}$ & 515,229 & 7,84012 & 559,758 & 7,21240 & 1997G & 1828,100 & 0,56511 & 1282,331 & 2,90409 \\
\hline 1994al & 755,092 & 5,27614 & 772,681 &, 13709 & $1997 \mathrm{H}$ & 95,405 & 3,83811 & 847,227 & \begin{tabular}{|l|}
4,70829 \\
\end{tabular} \\
\hline 1994am & 660,693 & 6,07376 & 510,505 & 7,91284 & 1997I & - & - & 353,183 & 11,43680 \\
\hline 1994an & 5,597 & 5,12401 & 672,977 & 95819 & 1997J & 1342,765 & 2,28041 & 1116,863 & 3,38049 \\
\hline $1995 \mathrm{aq}$ & 1004,616 & 3,54100 & 704,693 & 70879 & $1997 \mathrm{~K}$ & - & $-2,67464$ & 1224,616 & 2,78208 \\
\hline 1995ar & 1081,434 & 3,03856 & 972,747 & 3,81676 & 1997L & 1174,898 & 2,89569 & 972,747 & 2,78208 \\
\hline 1995as & 1288,250 & 1,59878 & 1066,596 & 3,33059 & $1997 \mathrm{~N}$ & - & - & 405,509 & 9,93353 \\
\hline 1995at & 51,962 & 3,71616 & 809,096 & 8 & 19970 & - &, 32 & 1282,331 & $-8,55733$ \\
\hline 1995aw & 31 & 5,79737 & 737,904 & 5,39574 & 1997P & $\mathbf{9 7 7 , 2 3 7}$ & 3,81094 & 928,966 & 4,12079 \\
\hline 1995ax & 1013,911 & 3,85961 & 772,681 & 5,21718 & 1997Q & 762,079 & 5,23052 & 737,904 & 5,42915 \\
\hline 1995ay & 912,011 & 4,25083 & 809,096 & 4,92632 & 1997R & 1361,445 & 2,34952 & 1770,109 & $-0,52256$ \\
\hline 1995az & 741,310 & 5,40765 & 1472,313 & $-4,78147$ & $1997 \mathrm{~S}$ & 1276,439 & 2,59689 & 1224,616 & 2,84963 \\
\hline 1995ba & \begin{tabular}{|l|}
790,678 \\
\end{tabular} & 4,93565 & \begin{tabular}{|l|}
772,681 \\
\end{tabular} & 5,08582 & $1997 \mathrm{ac}$ & 549,541 & 7,33840 & \begin{tabular}{|l|}
972,747 \\
\end{tabular} & 1,20870 \\
\hline $1996 \mathrm{cf}$ & 1051,962 & 3,61461 & 809,096 & 4,96594 & 1997af & 1158,777 & 3,08849 & \begin{tabular}{|l|}
672,977 \\
\end{tabular} & 6,00027 \\
\hline $1996 \mathrm{cg}$ & 972,747 & 3,89216 & 737,904 & 5,45015 & 1997ai & 859,014 & 4,54806 & 672,977 & 5,98805 \\
\hline 1996ci & 859,014 & 4,60767 & 672,977 & 5,99491 & 1997aj & 968,278 & 4,05794 & 1342,765 & 2,05331 \\
\hline $1996 \mathrm{ck}$ & 1207,814 & 3,03789 & 928,966 & 4,30142 & 1997am & 762,079 & 5,21609 & \begin{tabular}{||l|}
887,156 \\
\end{tabular} & 4,25366 \\
\hline $1996 \mathrm{cl}$ & 1986,095 & 0,22836 & 1169,499 & 3,35289 & 1997ap & 1706,082 & 1,52087 & 1614,359 & 1,86046 \\
\hline
\end{tabular}

In system (9) positive roots of the equations were considered only, and the received results have found out presence of dependences which according to report of calculations with allocation of meaning categories are presented by models in the form of position characteristics with instructions of an average absolute deviation (fig. 1):

$$
\begin{gathered}
k_{[9]}\left(\lg D_{L}\right)=41,1527664-12,45001197 \cdot \lg D_{L} \pm 0,1574237748, \\
k_{[17]}\left(\lg D_{L}\right)=45,4425535-13,8738728 \cdot \lg D_{L} \pm \mathbf{0 , 2 5 6 4 1 8 7 4 2 9 .}
\end{gathered}
$$

Thereby for cosmological distances scale the general analytical expression of settlement value for red shift take on form (fig. 1)

$$
Z_{\mathrm{K}}=\left(D_{L} / R_{0}\right) \cdot\left[1-\left(\mathrm{A}+\mathrm{B} \cdot \lg D_{L}\right) \cdot D_{L} / R_{0}\right]^{-1 /\left(\mathrm{A}+\mathrm{B} \cdot \lg D_{L}\right)} .
$$


For data [17] dependence (11) is practically linear and corresponds to fixed Hubble's constant $H_{0}=73,9 \mathrm{~km} \cdot \mathrm{c}^{-1} \cdot \mathrm{Mpc}^{-1}$. For data [9] dependence (11) in return time corresponds to change of Hubble's constant with 77,9 to $449,7 \mathrm{~km} \cdot \mathrm{c}^{-1} \cdot \mathrm{Mpc}^{-1}$ with smooth transition in present period to $76,4 \mathrm{~km} \cdot \mathrm{c}^{-1} \cdot \mathrm{Mpc}^{-1}$ (fig. 2).

To last case there corresponds an distances interval between local extreme of dependence (11) - [809,06; 897,84] Mpc. The nearest to it SN 1997Q and SN 1997P according to scale on the basis of parity (10) according to [9] will be accordingly on distances 2,64 and 3,18 billion light years. Further Hubble's constant is practically fixed.

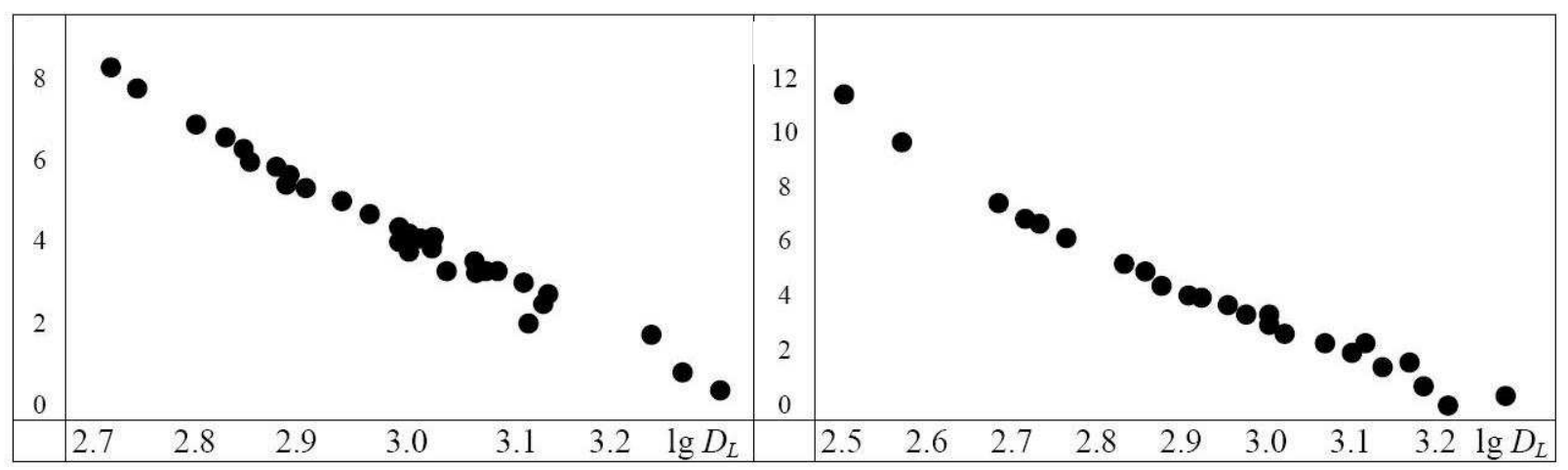

Fig. 1. Dependence of form parameter on photometric distance

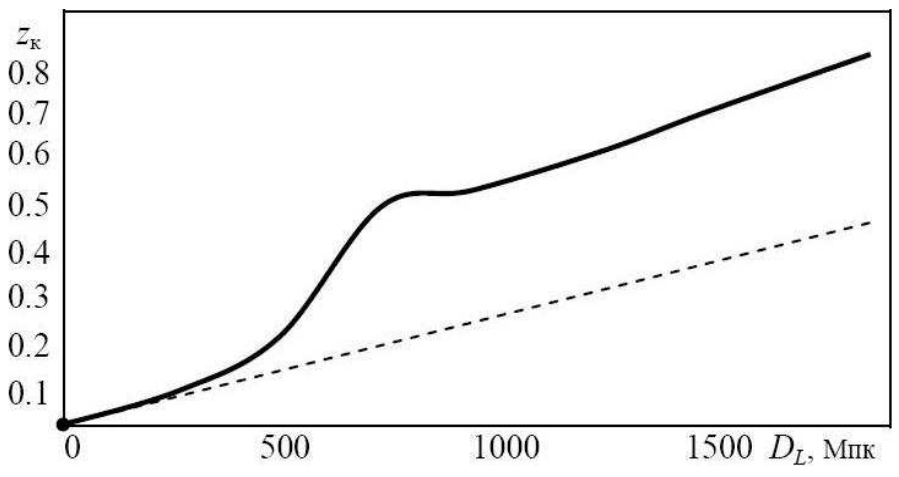

Fig. 2. Dependence of red shift on photometric distance for interpolation models (11)

\section{Physic, Statistics and Metrology}

Parametrical identification of components of substance with various constants of condition in model Friedman-Robertson-Walker on the basis of the photometric distances scale which reference points are supernovae type SN Ia in luminosity maximum, has been carried out in 1998-1999 by two ways. The group of researchers «High-Z SN Search Team» [8] used scale adjustment cosmological distances on the basis of red displacement under modules of distances of supernovae type SN Ia within the limits of Hubble's diagram, and group of researchers Supernova Cosmology Project [9] - adjustment of modeling dependence for maxima of luminosity supernovae under given measurements. Thus estimations of free parameters have appeared compatible, as it was necessary in a conclusion basis about «acceleration of expansion of the Universe». 
In these works the cosmological distances scale by results of calibration on reference points of photometric distances scale is not constructed. An problem essence that estimations of distance modules received thus, not speaking already about known extragalactic objects at $1<z<10$, for $z$ $\sim 0.97$ at $M_{\mathrm{st}} \mathrm{SN}$ Ia $=-19.5^{m}$ give the photometric distances exceeding Hubble radius. This circumstance has also purely metrological interpretation in terms of measuring problems theory [1].

The matter is that in cosmology it is considered not only a scale of photometric distances, and also scales of «angular distance», «accompanying distance» and «aberrational distance» which are connected with «Universe expansion». On distances of an order of 2 billion light years they practically coincide, and further - disperse.

For photometric distances scale, unlike other scales, the fundamental experimental fact is global Euclidean geometry for astronomical Universe. It allows considering as physical reality the «observable» distances which are not demanding for the interpretation cosmological models «extending Universe». As various modeling versions «Universe expansion» do not give the answer to type questions «and that was before», «why there was Big Bang», etc.

Developed in cosmology it is possible to compare situation to problem of gravitational waves when interpretation of century reduction for orbital period of double pulsar PSR 1913+16 within the limits of General relativity theory as consequences of gravitational waves radiation is considered in [18] as «the first experimental acknowledgement of their existence». However attempts of gravitational detection by method of direct measurement in projects LIGO (USA), «Virgo» (France, Italy), GEO-600 (Germany, Great Britain), TAMA-300 (Japan), LISA (international satellite project), «Nautilus» (Italy) and «Explorer» (Switzerland) while are unsuccessful [19].

In a considered problem used by the mentioned groups of researchers of model have the free parameters which physical sense has no experimental basis. On the same data the same problem has been solved with the help interpolation models cosmological red shift with form parameter. Dependence of parameter of the form on distance, and the beginning of «the accelerated expansion of Universe» was thus found out was displaced to red shift $z \approx 0,51$ or to the moment when «have blown up SN 1997P and SN 1997Q», i.e. about 3 billion years ago.

In basis interpolation models of red shift (8), unlike model Friedman-Robertson-Walker for an extending ideal homogeneous and isotropic liquid, mutual removal with initial speeds $0 \leq v$ $\leq c$ dot sources of electromagnetic radiation concentrated during the initial moment of time in small area some enough lays. For the observer in the centre of this area distribution of sources speeds on 
distance in «flat Universe» at expense of delay submits to the law $v \sim D \cdot\left(1 / H_{0}-D / c\right)^{-1}$, where $D-$ observable distance, $T_{0}=1 / H_{0}$ - time from the movement beginning moment [4]. For observable distances $D \ll c / H_{0}$ the law of speeds distribution becomes linear, further there is square-law amendment Hoyle [6], and on Hubble's radius the model has rupture of $2^{\text {nd }}$ sort.

Possible deviations from linear distribution of removal speeds of at Doppler interpretations for interpolation models are considered by form parameter. Check of correctness's has shown to parameterization, that this parameter is function of photometric distance, that essentially raises accuracy of interpreting model of red shift and, accordingly, calibrated in such a way cosmological distances scales on the basis of red shift. Certainly, anything surprising is not present that on the same data various models yield various results. Therefore received for interpolation models with form parameter results should be considered only as an illustration of calibration technique of cosmological distances scale for which more reliable data are necessary. After 4 objects that it is possible to consider as sign of statistical heterogeneity of data or their sign unequal accuracy have dropped out of sample in volume $42 \mathrm{SN}$ Ia.

Moreover, interpolation model of red shift according to Supernova Cosmology Project [9] has in a qualitative sense confirmed nonlinearity presence in dependence of red shift on distance, that at Doppler interpretation does not contradict a hypothesis about «acceleration of expansion of Universe». But for data from catalogue [17] for the same objects the same interpolation model has yielded negative result. From the point of view of Eljasberg-Hampel paradox [20, 21, 13], connected by that «statistical criteria cannot prove any hypothesis: they can specify only in «absence of a refutation»» [22], in the mathematical statistics negative results has greater weight.

However from the point of view of the theory of measuring problems [1] data Supernova Cosmology Project and [17] differ by data about effective star sizes on one category, i.e. by default, errors of data Supernova Cosmology Project 10 times less. And to it there should correspond more difficult model.

\section{Conclusion}

Thus, the phenomenon of change of dependence of red shift in spectra of extragalactic sources from observable distance is qualitative proves to be true. However questions on real accuracy of estimations of «accelerated expansion of Universe» at Doppler interpretation of red shift demand additional research and the comparative analysis of alternative models taking into account an inadequacy error. 


\section{References}

1. Gosudarstvennaja sistema obespechenija edinstva izmerenii. Opredelenie harakteristik matematicheskih modelej zavisimostej mezhdu fizicheskimi velichinami pri reshenii izmeritel'nyh zadach. Osnovnye polozhenija [State system for ensuring the uniformity of measurements. Determination of characteristics of mathematical models for dependences between physical values at the decision of measuring problems. Main principles]. (2000).

2. Burbidge G., Burbidge M. (1967). Quasi-stellar objects. S.-Francisco, London: Freeman \& Co.

3. Hubble E. (1929). A relation between distance and radial velocity among extragalactic nebulae. Proceedings NAS, Vol. 15, 168-173.

4. Levin S.F. (1980). Optimal'naja interpoljacionnaja fil'tracija statisticheskih harakteristik sluchajnyh funkcij $v$ determinirovannoj versii metoda Monte-Karlo $i$ zakon krasnogo smeshhenija [Optimum interpolation filtration of statistical characteristics for stochastic functions in determined version of Monte-Carlo method and the red shift law]. Moscow: Academy of sciences USSR.

5. Mattig W. (1958). Über den Zusammenhang zwischen Rotverschiebung und scheinbaren Helligkeit. Astronomischen Nachrichten, vol. 284, pp. 109-111.

6. Hoyle F. (1961). 44 ${ }^{\text {th }}$ Guthrie Lecture: Observational tests in Cosmology. Proceedings of Physical Society, part 1, 1-16.

7. Carroll S., Press W., Turner E. (1992). The Cosmological Constant. Annual Review of Astronomy \& Astrophysics, V.30, 499-542.

8. Riess A.G., Filippenko A.V., Challis P., Clocchiattia A., Diercks A., Garnavich P.M., Gilliland R.L., Hogan C.J., Jha S., Kirshner R.P., Leibundgut B., Phillips M.M., Reiss D., Schmidt B.P., Schommer R.A., Smith R.C., Spyromilio J., Christopher Stubbs, Nicholas B. Suntzeff, Tonry J. (1998). Observational evidence from supernovae for an accelerating universe and a cosmological constant. Astronomical journal Observational evidence from supernovae for an accelerating universe and a cosmological constant. Astronomical journal, vol. 116, 1009-1038.

9. Perlmutter S., Aldering G., Goldhaber G., Knop R.A., Nugent P., Castro P.G., Deustua S., Fabbro S., Goobar A., Groom D.E., Hook I.M., Kim A.G. (1999). Measurements of $\Omega$ and $\Lambda$ from 42 high-red shift supernovae. Astrophysical Journal, Vol. 517, 565-586.

10. Hinshaw G., Larson D., Komatsu E., Spergel D.N., Bennett C.L., Dunkley J., Nolta M.R., Halpern M., Hill R.S., Odegard N., Page L., Smith K.M., Weiland J.L., Gold B., Jarosik N., Kogut A., Limon M., Meyer S.S., Tucker G.S., Wollack E., Wrightet E. L. (2012). Nine-year 
Wilkinson microwave anisotropy probe observations: cosmological parameter results. Preprint WMAP.

11. Lang K.R. (1974). Astrophysical formulae. Part 2. Berlin, Heidelberg, N.Y.: Springer-Verlag.

12. Hamuy M., Phillips M.M., Suntzeff N.B., Robert A., Schommer R.A., Maza J., Avilés R. (1996). The Hubble diagram of the Calán/Tololo type Ia supernovae and value $\mathrm{H}_{0}$. Astronomical Journal, vol. 112, 2398-2429.

13. Levin S.F. (2014). Cosmological distances scale. Part I. «Unexpected» Results. Measurement Techniques, vol. 57, № 2, 117-122.

14. Levin S.F. (2012). Cosmological distances scale based on a red shift interpolation model. Measurement Techniques, vol. 55, № 6, 609-612.

15. Gosudarstvennaja sistema obespechenija edinstva izmerenij. Identifikacija raspredelenii verojatnostej pri reshenii izmeritel'nyh zadach [State system for ensuring the uniformity of measurements. Identification of probabilities distributions at the decision of measuring problems]. (2000).

16. Levin S.F. (2014). Cosmological distances scale. Part 3. Red shift Standards. Measurement Techniques, Vol. 57, № 9, 960-966.

17. Tsvetkov D.Yu., Pavlyuk N.N., Bartunov O.S., Pskovskii Yu.P. (2005). Supernovae Catalogue. Moscow: State Astronomical Sternberg Institute. Retrieved from: www.astronet.ru/db/sn/catalog.html.

18. Taylor J.H. jr. (1994). Dvojnye pul'sary i reljativistskoj gravitacii [Double pulsars and relativistic gravitation]. Uspekhi phizicheskikh nauk [Advances in Physical Sciences], vol. 164, № 7, 757-764.

19. Murzakhanov Z.G., Levin S.F., Belov I.Yu., Krivilev M.A., Sharnin L.M. (2011). The measurement problem of detecting gravitational waves from binary pulsars. Measurement Techniques, Vol. 54, № 11, 1225-1232.

20. Eljasberg P.E. (1983). Izmeritel'naja informacija: skol'ko ee nuzhno? kak ee obrabatyvat'? [The measuring information: how many it is necessary? How it to process?]. Moscow: Science.

21. Hampel F.R., Ronchetti E.M., Rousseeuw P.J., Stahel W.A. (1986). Robust Statistics: The Approach Based on Influence Functions. N.Y., Chichester, Brisbane, Toronto, Singapore: John Willey \& Sons Inc.

22. Korn G.A., Korn T.M. (1961). Mathematical Handbook for Scientists and Engineers. N.Y., Toronto, London: cMGRAW-Hill book company Inc. 